Review began 01/31/2022 Review ended 02/05/2022 Published 02/08/2022

(c) Copyright 2022

Al Hinai et al. This is an open access article distributed under the terms of the Creative Commons Attribution License CC-BY 4.0. which permits unrestricted use, distribution, and reproduction in any medium, provided the original author and source are credited.

\section{Pericardial Effusion in Erdheim-Chester Disease: A Case Report and a Mini Literature Review}

\author{
Juhaina Al Hinai ${ }^{1}$, Maitha Al Sibani ${ }^{1}$, Juhaina S. Al-Maqbali ${ }^{2}$, Abdullah M. Al Alawi ${ }^{3}$ \\ 1. Internal Medicine Training Program, Oman Medical Specialty Board, Muscat, OMN 2. Clinical Pharmacy, Sultan \\ Qaboos University Hospital, Muscat, OMN 3. Medicine, Sultan Qaboos University Hospital, Muscat, OMN
}

Corresponding author: Abdullah M. Al Alawi,dr.abdullahalalawi@gmail.com

\begin{abstract}
A 68-year-old man diagnosed with Erdheim-Chester disease presented to the emergency department with shortness of breath of one-day duration. Upon presentation, the patient was dyspnoeic and hypoxemic. The initial laboratory workup showed raised inflammation markers, and a chest $\mathrm{x}$-ray showed the presence of bilateral lung infiltrates; therefore, he was managed for community-acquired pneumonia with antimicrobial and other supportive measures. Due to lack of improvement, he had transthoracic echocardiography (ECHO), which showed a large pericardial effusion without tamponade. He was treated with corticosteroids and underwent pericardiocentesis, which resulted in remarkable symptomatic improvement. This case presents a serious manifestation of a rare disease and summarizes treatment options from the literature.
\end{abstract}

Categories: Cardiology, Internal Medicine, Hematology

Keywords: retroperitoneal fibrosis, pericardial tamponde, pericardial effusion, histiocytosis, erdheim-chester disease

\section{Introduction}

Erdheim-Chester disease (ECD) is a rare disease first described in 1930 and characterized by the presence of polymorphic granuloma infiltrated by foamy histiocyte and fibrosis [1, 2]. ECD manifestations include painful long bone osteosclerosis with endocrinopathy, interstitial lung disease, cutaneous manifestation neurodegenerative manifestations, and retroperitoneal fibrosis [2-4]. Also, ECD has serious cardiovascular manifestation coated aorta, coronary stenosis, myocardial infarction, pericarditis, and pericardial effusion with or without cardiac tamponade [2-4]. ECD's cardiovascular manifestations are common and associated with poor prognosis $[2,5]$. This case reports about a patient who presented with a large pericardial effusion. It also reviews the therapeutic approach based on the previously reported cases in the medical literature.

\section{Case Presentation}

A 68-year-old man presented to the emergency department at Sultan Qaboos University Hospital (SQUH) with a one-day history of worsening shortness of breath. He denied any fever, cough, or chest pain. His medical background included hypertension, chronic kidney disease, and ischemic heart disease that required percutaneous transluminal coronary angioplasty in 2009. In addition, he was diagnosed with ECD in 2018, after he presented with orthostatic hypotension due to newly introduced antihypertensives. During that admission, he was found to have lower lobe consolidation and nodules in the chest $\mathrm{x}$-ray, which triggered a series of investigations, that concluded a diagnosis of ECD with negative v-raf murine sarcoma viral oncogene homolog B1 (BRAF) mutation (Table 1). 


\section{Cureus}

\section{investigation}

High resolution computed tomography of the chest

Computed tomography of abdomen and pelvis

Magnetic resonance imaging of the brain

Positron emission tomography

Biopsy of lung nodules and left acetabular lytic lesion

Genetic mutations

Echocardiography
Results

Multiple small lung nodules (peri-lymphatic and sub-pleural) Atelectatic lung lesions Mild bilateral pleural effusion Small pericardial effusion

Multiple small mesenteric and para-aortic lymph nodes The left kidney is small with a simple cyst Right kidney normal in size, with few cysts

Few intracranial intra-axial lesions

FDG avid bilateral small lung nodules and nodular septal thickening with FDG avid atelectatic lung lesions FDG avid widespread bone lesions FDG avid brainstem lesion Focally increased FDG activity in the aortic wall at several levels

A fibroinflammatory process, with infiltration of histocytes, fibroblasts, foamy histocytes, and giant cells. Immunohistochemistry: Histocytes are positive for Vimentin and CD68 and are negative for Pan-CK, and CD1a Ki67 is very low

BRAF $^{\mathrm{V} 600 \mathrm{E}}$ and NRAS are not detected

Ejection fraction $50-70 \%$ with good contractility. No valvular abnormality. Minimal posterior and lateral pericardial effusion measuring $0.6 \mathrm{~cm}$, without tamponade effect.

\section{TABLE 1: Diagnostic workup leading to the diagnosis of Erdheim-Chester disease in 2018}

FDG: fluorodeoxyglucose, Pan-CK: pan Cytokeratin antibodies, CD1a: Cluster of Differentiation1a, BRAF: v-raf murine sarcoma viral oncogene homolog B1, NRAS: neuroblastoma rat sarcoma virus,

ECD management included six weekly of injection infliximab, oral azathioprine $50 \mathrm{mg}$ (two times per day), and prednisolone (5 $\mathrm{mg}$ daily), and his condition remained stable until his recent presentation to the emergency department.

Upon presentation, his vitals were as follows: temperature 37.9 centigrade, heart rate 90 beats per minute, respiratory rate 32 breaths per minute, blood pressure 193/100 $\mathrm{mm} \mathrm{Hg}$, oxygen saturation 87\% on ambient air. On examination, he was in distress, with significant crepitation all over his chest but no pedal edema. His inflammatory markers were high, and chest x-ray showed bilateral reticulonodular opacities, increased vascular markings, and bilateral pleural effusion. COVID-19 RNA was not detected, and a workup for atypical pneumonia was done (Table 2). 


\section{Cureus}

\begin{tabular}{|c|c|c|}
\hline Haemoglobin (g/dL) & 10.4 & 11.5-15.5 \\
\hline White blood cells count $\left(x 10^{9} / \mathrm{L}\right)$ & 11.7 & $2.2-9.0$ \\
\hline Platelet $\left(x 10^{9} / \mathrm{L}\right)$ & 480 & $150-450$ \\
\hline C-reactive protein (mg/L) & 112 & $0-10$ \\
\hline Serial troponin (ng/L) & $88-57-45$ & $<14$ \\
\hline Sodium (mmol/L) & 136 & 135-145 \\
\hline Potassium (mmol/L) & 4.9 & $3.5-5.1$ \\
\hline Bicarbonate $(\mathrm{mmol} / \mathrm{L})$ & 19 & $21.8-26.9$ \\
\hline Chloride (mmol/L) & 100 & 98-107 \\
\hline Magnesium (mmol/L) & 0.72 & $0.66-0.89$ \\
\hline Corrected calcium (mmol/L) & 2.42 & $2.15-2.55$ \\
\hline Phosphate (mmol/L) & 1.24 & $0.81-1.45$ \\
\hline Creatinine (umol/L) & 188 & $59-104$ \\
\hline Estimated GFR $\left(\mathrm{mL} / \mathrm{min} / 1.73 \mathrm{~m}^{2}\right)$ & 31 & $>90$ \\
\hline Urea (mmol/L) & 7.9 & $2.8-8.1$ \\
\hline Anion gap (mmol/L) & 17 & $5-13$ \\
\hline
\end{tabular}

\section{TABLE 2: Laboratory tests results upon presentation to the hospital}

He was treated with antimicrobials (IV ceftriaxone, oral azithromycin, and oseltamivir) and an $\mathrm{O} 2$ supplement. He had transthoracic echocardiography, which demonstrated a large pericardial effusion measuring $2.7 \mathrm{~cm}$ posteriorly, without tamponade effect (Figure 1).
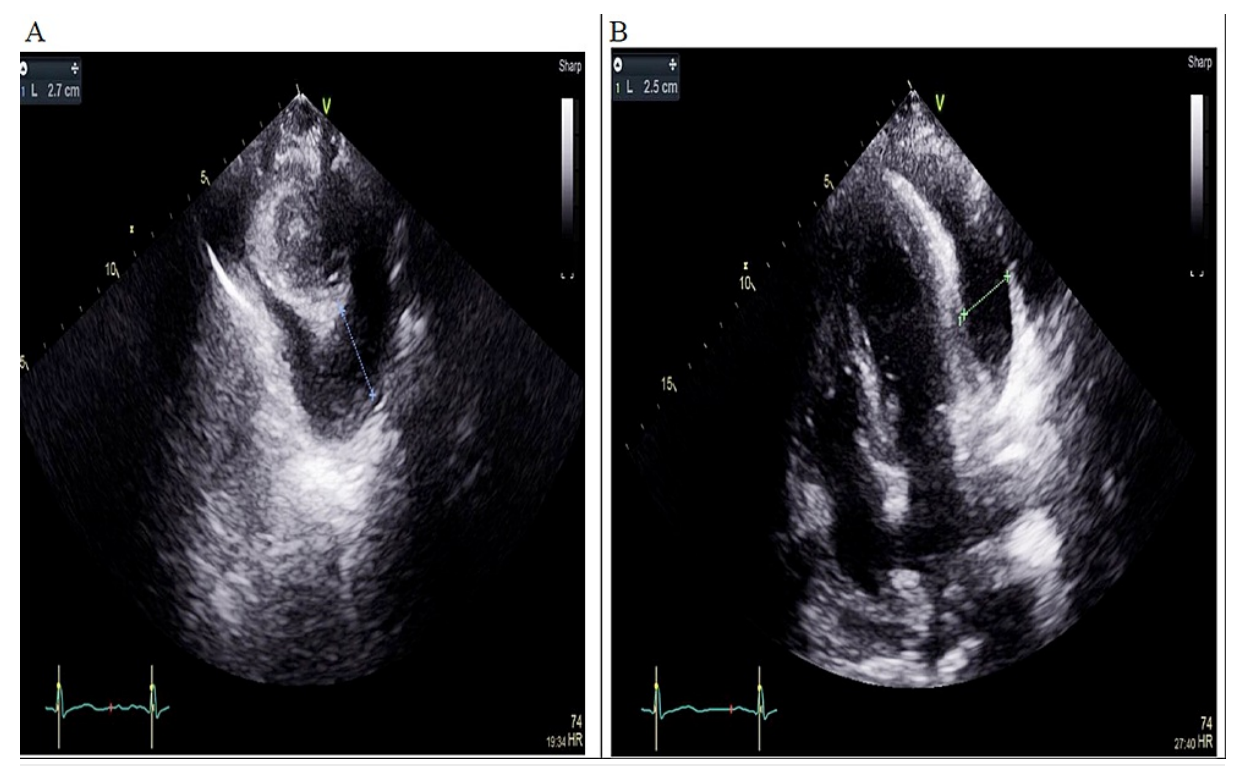

FIGURE 1: Parasternal short axis window (A) and apical 4 chamber window show large pericardial effusion (B).

He was treated with 1 gram of methylprednisolone for three days, followed by ultrasound-guided 
percutaneous pericardiocentesis due to ongoing symptoms. A round $500 \mathrm{ml}$ of straw-colored pericardial fluid was drained and analysis showed leukocytosis and erythrocytes with no bacterial growth from culture. In addition, cytology revealed high mesothelial cells and macrophages that could not be distinguished from normal macrophages (non-Langerhans cells).

After the pericardiocentesis, the patient had symptomatic improvement accompanied by improvement in his oxygen requirement during the hospital stay. Therefore, he was discharged with $50 \mathrm{mg}$ of prednisolone (to be tapered by $5 \mathrm{mg}$ every week), and he was booked to be reviewed in an outpatient clinic.

\section{Discussion}

ECD is an extremely rare disease of non-Langerhans cell histiocytosis; it was first described by William Chester and Jakob Erdheim and characterized by uncontrolled chronic inflammation [2, 6, 7]. The oncogenic BRAF (V600E) mutation was reported in $54 \%$ of the cases $[5,6,8]$. The disease progress through fibroinflammatory infiltrates to tissue organ by the presence of foamy histiocytes, multinucleated giant cells, and lymphocytes $[6,9]$. The manifestations of ECD in this patient at the time of diagnosis included the presence of lesions in the brain stem, lung, bones, para-aortic lung nodules, and evidence of minimal pericardial effusion. Overall, cardiac involvements are present in $75 \%$ of patients with ECD, and it is associated with worsening disease prognosis leading to death in around $60 \%$ of the patients. The most common cardiac involvement is pericardial effusion, leading to tamponade [10]. The only treatment option approved by FDA in 2017 for ECD patients with positive BRAF (V600E) mutation is BRAF kinase inhibitor (oral vemurafenib) $[11,12]$.

On the other hand, a literature review revealed various immunosuppressive treatments used to treat patients with negative mutation BRAF (V600E). These treatments included interferon alfa, steroids, methotrexate, infliximab, or oral azathioprine as monotherapy or in combination, as described by several case reports $[8,13]$. ECD prognosis is affected by the type of organ involved. For example, central nervous system defects, which occur mainly in older patients and cardiovascular lesions, are associated with poor prognosis, yet the prognosis varies provided that patients get supportive care as early as possible [14]. To date, there are 31 case reports and four case series published in the literature describing the management of pericardial effusion in patients with ECD. Percutaneous pericardiocentesis is the most common intervention used to remove pericardial effusion, while the pericardial window is used only in case of recurrence $[4,7,15]$. Nevertheless, interferon alfa is considered the first-line option for management of ECD, for its associated improvement in patients' five-year survival $(82.7 \%)[13,14]$.

\section{Conclusions}

ECD is a non-Langerhans is a rare cell histiocytosis that involves fibro-inflammatory effects associated with multi-organ involvements. Pericardial effusion is a severe manifestation of ECD. We described a successful therapeutic approach that included systematic steroids and pericardiocentesis to treat a large pericardial effusion.

\section{Additional Information \\ Disclosures}

Human subjects: Consent was obtained or waived by all participants in this study. Conflicts of interest: In compliance with the ICMJE uniform disclosure form, all authors declare the following: Payment/services info: All authors have declared that no financial support was received from any organization for the submitted work. Financial relationships: All authors have declared that they have no financial relationships at present or within the previous three years with any organizations that might have an interest in the submitted work. Other relationships: All authors have declared that there are no other relationships or activities that could appear to have influenced the submitted work.

\section{References}

1. Papo M, Emile JF, Maciel TT, et al.: Erdheim-Chester disease: a concise review. Curr Rheumatol Rep. 2019, 21:66. 10.1007/s11926-019-0865-2

2. Haroche J, Cohen-Aubart F, Amoura Z: Erdheim-Chester disease. Blood. 2020, 135:1311-8. 10.1182/blood.2019002766

3. Mazor RD, Manevich-Mazor M, Kesler A, et al.: Clinical considerations and key issues in the management of patients with Erdheim-Chester Disease: a seven case series. BMC Med. 2014, 12:221. 10.1186/s12916-0140221-3

4. Sanchez-Nadales A, Anampa-Guzman A, Navarro-Motta J: Erdheim-Chester disease with extensive pericardial involvement: a case report and systematic review. Cardiol Res. 2020, 11:118-28. 10.14740/cr1025

5. Nicolazzi MA, Carnicelli A, Fuorlo M, Favuzzi AM, Landolfi R: Cardiovascular involvement in ErdheimChester disease: a case report and review of the literature. Medicine (Baltimore). 2015, 94:e1365. 10.1097/MD.0000000000001365

6. Alexiev BA, Staats PN: Erdheim-Chester disease with prominent pericardial effusion: cytologic findings and review of the literature. Diagn Cytopathol. 2014, 42:530-4. 10.1002/dc.22957

7. Oliveira M, Monteiro S, Dos Santos J, Silva AC, Morais Ferreira R: Erdheim-Chester disease: a rare clinical 


\section{Cureus}

entity. Eur J Case Rep Intern Med. 2020, 7:001630. 10.12890/2020 001630

8. Haroche J, Charlotte F, Arnaud L, et al.: High prevalence of BRAF V600E mutations in Erdheim-Chester disease but not in other non-Langerhans cell histiocytoses. Blood. 2012, 120:2700-3. 10.1182/blood-201205-430140

9. Haroche J, Amoura Z, Dion E, et al.: Cardiovascular involvement, an overlooked feature of Erdheim-Chester disease: report of 6 new cases and a literature review. Medicine (Baltimore). 2004, 83:371-92. 10.1097/01.md.0000145368.17934.91

10. de Miguel Criado J, Aguilera Del Hoyo LF, García Del Salto L, et al.: Case 224: Cardiac Involvement in Erdheim-Chester Disease. Radiology. 2015, 277:916-21. 10.1148/radiol.2015131751

11. Oneal PA, Kwitkowski V, Luo L, et al.: FDA approval summary: vemurafenib for the treatment of patients with Erdheim-Chester disease with the BRAFV600 mutation. Oncologist. 2018, 23:1520-4 10.1634/theoncologist.2018-0295

12. Diamond EL, Subbiah V, Lockhart AC, et al.: Vemurafenib for BRAF V600-mutant Erdheim-Chester disease and Langerhans cell histiocytosis: analysis of data from the histology-independent, phase 2, open-label VEBASKET study. JAMA Oncol. 2018, 4:384-8. 10.1001/jamaoncol.2017.5029

13. Arnaud L, Hervier B, Néel A, et al.: CNS involvement and treatment with interferon- $\alpha$ are independent prognostic factors in Erdheim-Chester disease: a multicenter survival analysis of 53 patients. Blood. 2011, 117:2778-82. 10.1182/blood-2010-06-294108

14. Toya T, Ogura M, Toyama K, et al.: Prognostic factors of Erdheim-Chester disease: a nationwide survey in Japan. Haematologica. 2018, 103:1815-24. 10.3324/haematol.2018.190728

15. Lin J, Tang SQ, Chen JC, Zhang YH, Zhang Q: Erdheim-Chester disease: an unusual etiology of recurrent massive pericardial effusion. Can J Cardiol. 2020, 36:1832.e11-3. 10.1016/j.cjca.2020.04.040 